\section{SARS-CoV-2 B.1.1.7 Variant Infection in Malayan Tigers, Virginia, USA}

\author{
Patrick K. Mitchell, Mathias Martins, Tara Reilly, \\ Leonardo C. Caserta, Renee R. Anderson, \\ Brittany D. Cronk, Julia Murphy, Erin L. Goodrich, \\ Diego G. Diel
}

Author affiliations: Cornell University, Ithaca, New York, USA (P.K. Mitchell, M. Martins, L.C. Caserta, R.R. Anderson, B.D. Cronk, E.L. Goodrich, D.G. Diel); Virginia Zoo, Norfolk, Virginia, USA (T. Reilly); Virginia Department of Health, Richmond, Virginia (J. Murphy)

DOI: https://doi.org/10.3201/eid2712.211234

We report infection of 3 Malayan tigers with severe acute respiratory syndrome coronavirus 2 (SARS-CoV-2) B.1.1.7 (Alpha) variant at a zoologic park in Virginia, USA. All tigers exhibited respiratory signs consistent with SARSCoV-2 infection. These findings show that tigers are susceptible to infection with the SARS-CoV-2 B.1.1.7 variant.

$\mathrm{O}$ April 4, 2021, a 5-year-old male Malayan tiger (Panthera tigris jacksoni) at the Virginia Zoo (Norfolk, VA, USA) began exhibiting lethargy, labored breathing, coughing, intermittent upper respiratory sounds, hyporexia, and mucoid nasal discharge. On April 7, another 5-year-old male Malayan tiger began experiencing labored breathing, cough, clear nasal discharge, and hyporexia. On April 10, a third Malayan tiger, a 10-year-old male, had cough and later clear nasal discharge. The tigers' clinical signs resolved by April 15, eleven days after the outbreak began.

Zoo staff collected nasal swab and fecal samples from the 5-year-old tigers on April 9 and the 10-yearold tiger on April 13 and submitted these to Cornell University's Animal Health Diagnostic Center (AHDC; Ithaca, NY, USA). AHDC tested samples for Bordetella sp., Chlamydia felis, Mycoplasma cynos, M. felis, Streptococcus equi subspecies zooepidemicus, influenza virus, pneumovirus, feline calicivirus, and feline herpesvirus; all results were negative. All samples tested positive for severe acute respiratory syndrome coronavirus 2 (SARS-CoV-2) by EZ-SARS-CoV-2 Real-Time RT-PCR Test (Tetracore, Inc., https://tetracore.com). We isolated SARS-CoV-2 from respiratory and fecal specimens from the first tiger. Testing at the US Department of Agriculture National Veterinary Services Laboratories (Ames, IA, USA) confirmed SARS-CoV-2 infection. We screened the tiger samples using TaqPath COVID-19 RT-PCR Kit (Thermo
Fisher Scientific, https://www.thermofisher.com), which revealed a spike gene dropout in samples from all 3 tigers; only the nucleoprotein and open reading frame 1 ab gene targets were detected, suggesting B.1.1.7 variant infection.

We performed whole-genome sequencing on all samples by using MinION (Oxford Nanopore Technologies, https://nanoporetech.com), as previously described (1). We assembled reads using the ARTIC ncov-2019 protocol (ARTIC Network, https://artic. network) and Medaka (Oxford Nanopore Technologies) for variant calling. We obtained near-complete (29,702-29,710-bp) assemblies from all nasal swab specimens (GenBank accession nos. MZ305031-3) but no assemblies from fecal samples. We identified respiratory specimen genomes as lineage B.1.1.7 (Alpha variant) by using Pangolin version 2.4.2 (https:/ / github.com/ cov-lineages/pangolin). We used Nextstrain (https:/ / nextstrain.org) for phylogenetic analysis of tiger-derived sequences and other B.1.1.7 sequences downloaded from GISAID (https://www.gisaid.org) on April 15, 2021 (2,3). Tiger-derived sequences all were identical, except 1 manually corrected homopolymer repeat error, and fell into a clade defined by a C4900T mutation containing other samples collected primarily in the United States. Tiger-derived sequences differed from others in the clade by 1 single-nucleotide polymorphism in the spike gene (K558N) (Figure, panel A). Using the vdb tool (4), we found 46 additional B.1.1.7 sequences that had the K558N mutation in GISAID on July 22, 2021; all were collected from Virginia during March 27-July 7, 2021. However, phylogenetic analysis of these sequences and the tiger-derived sequences showed divergence of 11 single-nucleotide polymorphism, minus the divergence producing the $\mathrm{K} 558 \mathrm{~N}$ mutation (Figure, panel B), indicating the sequences are not related epidemiologically.

The source of the tigers' infection is unknown. The zoo has been open to the public, but transmission from a visitor is unlikely because tiger exhibit areas are separated from visitors by either a glass enclosure or $\geq 9 \mathrm{~m}$ distance. The most plausible explanation is that $\geq 1$ tiger acquired the virus from a keeper because they had close contact. However, no employees tested positive for SARS-CoV-2 nor had symptoms during the 4 weeks before the tigers' symptom onset. Nine keepers were responsible for the animals' daily care; 2 other persons prepared animal diets daily. Employees were required to wear facemasks always, indoors and outdoors; everyone wore standard 2-ply surgical masks or homemade cloth facemasks. Staff also were required to wear gloves when handling and preparing food and when servicing animal areas. Furthermore, staff were 

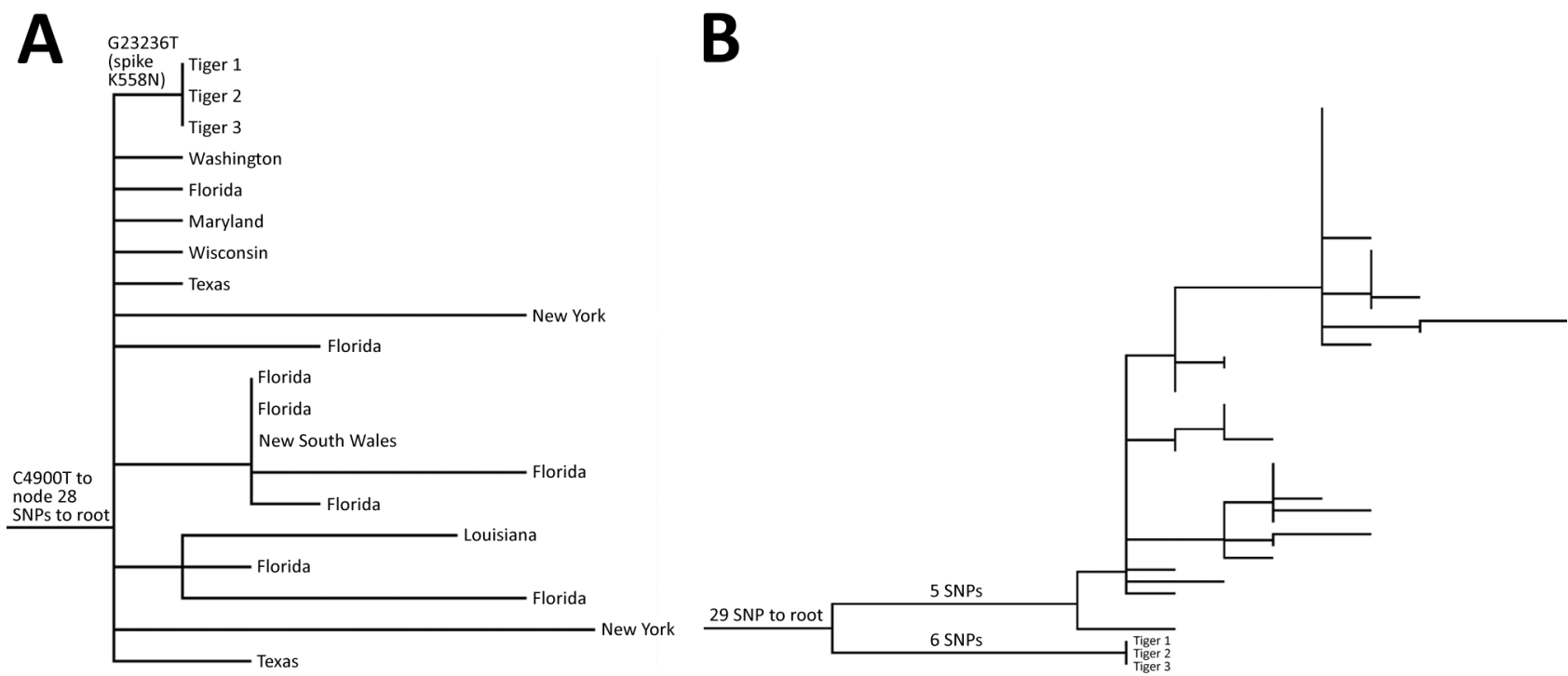

Figure. Maximum-likelihood phylogenetic trees of severe acute respiratory syndrome coronavirus 2 from 3 Malayan tigers, Virginia, USA. Tiger samples are numbered in order of symptom onset. A) Subset of phylogenetic tree showing parent (G23236T) and grandparent (C4900T) nodes of the tiger sequences, with tips labeled as states of origin in the United States or Australia. B) Phylogenetic tree showing that other B.1.1.7 viruses detected in Virginia that contain the K558N mutation are not epidemiologically related to the sequences detected in tigers 1,2 , and 3 . SNP, single-nucleotide polymorphism.

required to step into an accelerated hydrogen peroxide disinfectant footbath when entering the tiger building and diet kitchen. The 3 tigers might have been infected by an employee, or 1 tiger was infected, then transmission occurred to the others. Two tigers lived in the same enclosure and had no direct contact with the third, but all 3 rotated through common enclosure spaces.

After identification of the tiger infections, 4 additional zoo animals were tested: 1 lion (Panthera leo) with lethargy and hyporexia $\approx 1$ week after SARS-CoV-2 diagnosis in the tigers; another asymptomatic lion because of age and proximity to the first lion; and 2 degus (Octodon degus) that died in late March and had interstitial pneumonia on necropsy. AHDC tested nasal swab samples from the lions and frozen spleen and cecum samples from the degus by reverse transcription PCR; all results were negative for SARS-CoV-2.

Our findings underscore felid susceptibility to SARS-CoV-2, which also has been detected in captive snow leopards (Panthera uncia) and pumas (Puma concolor) (5). Other nonhuman species, including gorillas (Gorilla gorilla), minks (Neovison vison), and ferrets (Mustela putorius furo), have acquired SARS-CoV-2; additional species have been shown to be susceptible experimentally (5-7). Domestic cats and dogs in the United Kingdom and United States reportedly had B.1.1.7 infections, suggesting that mutations characterizing this lineage are not constrained to a host range (8; L. Ferasin et al., unpub. data, https://doi.org/10.1101/2021.03.18.435945).
Monitoring animals for SARS-CoV-2 infection is critical to determining potential host range, particularly as new virus variants emerge and spread.

This article was preprinted at https://doi.org/10.21203/ rs.3.rs-618448/v1.

\section{Acknowledgments}

We gratefully acknowledge the originating and submitting laboratories of the sequence data submitted to GISAID (https://www.gisaid.org) used in this manuscript.

The sequencing infrastructure at the Animal Health Diagnostic Center, Cornell University, used in this study, is funded through grants from the National Animal Health Network to D.G.D. (grant nos. AP20VSD and B000C020).

\section{About the Author}

Dr. Mitchell is a research associate in the Department of Population Medicine and Diagnostic Sciences at Cornell University. His primary research interest is molecular epidemiology of infectious diseases.

\section{References}

1. Caserta LC, Mitchell PK, Plocharczyk E, Diel DG. Identification of a SARS-CoV-2 lineage B1.1.7 virus in New York following return travel from the United Kingdom. Microbiol Resour Announc. 2021;10:e00097-21. https://doi.org/10.1128/MRA.00097-21 
2. Hadfield J, Megill C, Bell SM, Huddleston J, Potter B, Callender C, et al. Nextstrain: real-time tracking of pathogen evolution. Bioinformatics. 2018;34:4121-3. https:/ / doi.org/10.1093/bioinformatics/bty407

3. Sagulenko P, Puller V, Neher RA. TreeTime: maximumlikelihood phylodynamic analysis. Virus Evol. 2018;4:vex042. https://doi.org/10.1093/ve/vex042

4. West AP, Wertheim JO, Wang JC, Vasylyeva TI, Havens JL, Chowdhury MA, et al. Detection and characterization of the SARS-CoV-2 lineage B.1.526 in New York. Nat Comm. 2021;12:4886. https://doi/org/10.1038/s41467-021-25168-4

5. World Organisation for Animal Health. COVID-19 [cited 2021 May 19]. https:/ / www.oie.int/en/what-we-offer/ emergency-and-resilience/covid-19

6. US Department of Agriculture Animal and Plant Health Inspection Service. Cases of SARS-CoV-2 in animals in the United States [cited 2021 May 19]. https:/ / www.aphis.usda. gov/aphis/dashboards/tableau/sars-dashboard

7. Delahay RJ, de la Fuente J, Smith GC, Sharun K, Snary EL, Flores Girón L, et al. Assessing the risks of SARS-CoV-2 in wildlife. One Health Outlook. 2021;3:7. https://doi. org/10.1186/s42522-021-00039-6

8. Hamer SA, Ghai RR, Zecca IB, Auckland LD, Roundy CM, Davila E, et al. SARS-CoV-2 B.1.1.7 variant of concern detected in a pet dog and cat after exposure to a person with COVID-19, USA. Transbound Emerg Dis. 2021 May 12 [Epub ahead of print] https:// doi.org/10.1111/tbed.14122

Address for correspondence: Diego G. Diel, Animal Health Diagnostic Center, Cornell University, 240 Farrier Rd, Ithaca, NY 14853, USA; email: dgdiel@cornell.edu

\section{Postmortem Stability of SARS-CoV-2 in Mouse Lung Tissue}

\author{
Sophie A. Valkenburg, Samuel M.S. Cheng, \\ Asmaa Hachim, Malik Peiris, John Nicholls \\ Author affiliations: The University of Hong Kong, Hong Kong, China
}

DOI: https://doi.org/10.3201/eid2712.211621

The infectivity of severe acute respiratory syndrome coronavirus 2 in deceased persons and organisms remains unclear. We studied transgenic K18 hACE2 mice to determine the kinetics of virus infectivity after host death. Five days after death, virus infectivity in the lung declined by $>96 \%$ and RNA copies declined by $48.2 \%$.
The safe handling and disposal of bodies of persons who have died of coronavirus disease (COVID-19) is vital for infection control. Although cremation or burial practices are mainly dictated by religious and societal customs, deaths associated with contagious illness warrant appropriate precautions. Severe acute respiratory syndrome coronavirus 2 (SARS-CoV-2), the causative agent of COVID-19, is rapidly inactivated $\left(>2 \log _{10}\right)$ within hours on nonporous surfaces (1). In addition, several studies have detected viral RNA by reverse transcription PCR (RT-PCR) of nasopharyngeal and pharyngeal mucosal swab specimens, skin swab specimens, and tissue samples collected during autopsies at different times after death (2-5). Furthermore, infectious virus was isolated in 2 of 4 cases at 4-17 days postmortem; however, this study did not quantify virus titers to determine the loss of virus infectivity (6). A separate study found that infectious virus was undetectable after exhumation at 3-4 months postmortem (7). Overall, RNA detection by RT-PCR might not directly correlate with virus infectivity or duration of symptomatic disease.

Transgenic K18-hACE2 mice provide a surrogate model to study the kinetics of SARS-CoV-2 viral replication during infection (8) and after host death. In humans and K18-hACE2 mice, little evidence exists for extrapulmonary dissemination of SARS-CoV-2, except for neurotropism in younger mice, a finding that has not been demonstrated reliably in humans. We investigated the temporal decay of infectious SARSCoV-2 in postmortem tissues of infected K18-hACE2 mice. All experimental procedures were conducted in accordance with the standards and approved by the Committee on the Use of Live Animals in Teaching and Research (approval no. 5511-20) at The University of Hong Kong (Hong Kong, China).

We infected twelve 14-20-week-old mice with $1 \times$ $10^{4} 50 \%$ tissue culture infectious dose $\left(\mathrm{TCID}_{50}\right) / 25 \mu \mathrm{L}$ SARS-CoV-2 by the intranasal route. Five days later, after the mice had lost 18.8\% (SD 7.77\%) of their body weight, we euthanized them by ketamine/xylazil anesthesia. We wrapped each carcass in a sealable plastic bag, similar to the storage of human corpses, and stored them intact at $4^{\circ} \mathrm{C}$, which is standard mortuary temperature. On days $0,1,5$, and 14 after death, we dissected 3 carcasses and tested the lung tissue for coronavirus nucleoprotein $(\mathrm{N})$ by histologic and immunohistochemistry assays (9) (Appendix Figure, panels A-H, https://wwwnc.cdc.gov/EID/ article/27/12/21-1612-App1.pdf). We quantified infectious virus by culture (Figure, panel A) and viral RNA by RT-PCR (Figure, panel B) (Appendix). 